\title{
PROPUESTAS PARA UN DIALOGO \\ SOBRE EL ESPACIO LITERARIO BOLIVIANO
}

POR

\author{
BLANCA WIETHÜCHTER \\ Universidad Mayor de San Andrés, La Paz
}

Si toda lengua transporta, además de lo que quiere comunicar, un modo, una forma, en una palabra, un espíritu, éste explica la manera de habitar un medio, sugiere la relación con la sociedad y con la naturaleza. El espíritu de una lengua es producto de una selección asimilativa, de una adecuación a lo vivido. Es, en resumen, la representación del mundo de una cultura. Finalmente, podría decirse que el lenguaje es la práctica de una memoria particular que puede entenderse como un espesor. De ese modo, el lenguaje, la palabra y, finalmente, la literatura implican, además de lo que dicen, una serie de otros sentidos que permiten re-conocer, reconocer-se, recordar el mundo al cual se adscriben.

¿Qué sucede si este lenguaje es transportado a otro mundo, a otra naturaleza sobre la cual tiende a sobreimprimirse, puesto que es un espacio habitado y, por tanto, nombrado? El poder y la violencia reprimen y someten, pero no pueden impedir que lo nombrado sea «desnombrado», y la historia de los pueblos andinos lo demuestra a través de cuatro siglos de dominación.

Ahora bien, al lograr Bolivia su independencia como país (1825), éste asume una homogeneidad puramente nominal, la misma que sólo pudo ser definida en tanto que proyecto de la elite criolla. Sus habitantes no hablan el mismo idioma; el «español», en tanto idioma oficial (hacia 1925 sólo lo habla un 20 por 100 de la población), es utilizado en todo lo que hace al poder: el gobierno, las funciones legislativas y judiciales, en el comercio, es decir, en todos los ámbitos que son regidos por los grupos dominantes. Por el contrario, el lenguaje mayoritario, el de la tierra, será un lenguaje recluido en lo doméstico, en los ámbitos del trabajo, es decir, en los espacios en que se refugiarán y resistirán los grupos étnicos nativos, la cultura de los dominados. 
El idioma español, marcado por el signo del poder, de la conquista, será, a mitad del siglo pasado, el signo de un vigor, de una fuerza en la lucha por imponerse. Y una muestra de ello bien podría ser lo que los críticos han considerado la mejor novela boliviana: Juan de la Rosa, de Nataniel Aguirre. Tal vez una de las pocas novelas que pueden considerarse de «fundación», en la medida en que se relata a través de la mirada de un niño (¿la naciente Bolivia?) los acontecimientos de la guerra por la independencia.

Después de esta conquista, la lengua española, que transporta otra memoria, referida a una nominación occidental que relata otra experiencia vital, se hace en la literatura, valga la reiteración, «literaria».

El romanticismo de final del siglo xIX es parte de un diálogo libresco, tal como lo es también el modernismo. El lenguaje trabajado en dicha literatura no forma parte de una experiencia vital, sino que se enraíza en otro espacio vivido y legitimado como tradición y cultura en oposición a la «barbarie». Cuando la literatura intenta pisar suelo propio se hace manifiesta la contradicción entre lo real y lo nombrado. Un lenguaje no apto para encarnar esa realidad y que comienza por acudir al nombre natural por necesidad, sea quechua, aymara o guaraní, para traducir lo real, pero que en el contexto lingüístico resulta exótico y muchas veces peca de un costumbrismo excesivo, una mimesis que se reduce a los límites de su espacio.

Pienso que después de la guerra del Pacífico (1879) y años después la guerra del Chaco (1933-1936), el proyecto de nación boliviana se convierte, con matices diferentes después de cada acontecimiento bélico, en el objetivo fundamental de los grupos gobernantes. A este proyecto corresponde el de una literatura boliviana que asume como una de sus tareas fundamentales la de nominar esa nación, nominación que hace sólo pocos años se explicita en intenciones escriturales como las ensayadas por Augusto Céspedes y Pedro Shimose.

Como punto de partida, creo que la literatura boliviana debe ser situada, primero, como proyecto de fundación de una elite criolla ligado a la formación de una nación que se quiere autónoma (no tenemos sino ciento sesenta años de independencia), y segundo, que este proyecto no puede. sino entenderse como un polo de la dicotomía criollo-indígena. Que esta polarización está sujeta a una notoria contradicción, que, además de manifestarse en la literatura criolla, se expresa en la literatura oral indígena. En ésta el polo «blanco» es vivido simétricamente como anticultura (Blanca Wiethüchter, «El guerrero aymara», en Hipótesis, La Paz, 1984, pp. 41$62)$, lo que de algún modo expresa la derrota de una conquista que se quiso totalizadora, en la medida en que el mundo andino es pensado por 
los indígenas respecto al criollo o blanco como dador de orden y sentido, y al otro, al criollo, como no podía ser de otro modo, como un mundo inmerso en lo gratuito del poder y la violencia.

E1 proyecto literario boliviano, desde sus inicios, está, pues, signado a dar cuenta de complejas relaciones sociales (pienso en José María Arguedas, que al no lograr superar la tensión de este dualismo - lo sabemos todos- se suicida). ¿Y no es ninguna sorpresa que en gran parte de los textos actuales se introduzcan experiencias similares a la de Arguedas con un trabajo sobre estructuras y formas indígenas, tal vez prefigurando así un modo «boliviano» de expresión, una memoria?

Ahora bien, intento en este trabajo, aunque de modo breve, diseñar algunos puntos en la curva que sigue el lenguaje en este proyecto de literatura boliviana en sus formas literarias, los que pueden ser considerados como pistas para un futuro diálogo. En este sentido, este trabajo debe ser considerado como un primer avance de ese diálogo.

Concebir la literatura como un proyecto de nominación será, pues, uno de los fundamentos de este diseño. Perspectiva que, además, se ve confirmada por la historia a través de un proyecto de formación de la «conciencia nacional», que remata, finalmente, en la revolución de 1952 en términos de nacionalismo: la concepción de un Estado como ente unificador, integrador de la nación. Es a partir de esta revolución que los críticos -aunque no inmediatamente después- señalan la existencia de una nueva literatura.

De las obras que realizan este proyecto -evidentemente se trata de un cuerpo literario del que sólo tomo algunos de sus componentes-intentaré ordenarlas por el gesto que expresan. Así, he de hablar de escritura de acontecimiento y de una literatura emancipada como las dos posibles vertientes de la literatura actual. (No creemos en las clasificaciones sino como ordenadores de un discurso.)

Los tres novelistas de principios de siglo, Chirveches, Arguedas y Mendoza, pueden ser considerados como los fundadores de los territorios sobre los que una y otra vez volverán los escritores: el pueblo/ciudad, el campo y las minas. Pero también el gesto testimonial de sus obras -el fraude electoral, la vida en las minas, la violencia y la injusticia en el campo- es índice de un continuo y reiterado referente. La literatura que se expresa en esos términos - referenciales- sería la literatura de acontecimiento, y que, no cabe duda, nutre intensivamente nuestro espacio literario.

En esta literatura, la palabra se hace excusa para la descripción, «instrumento», de tal modo que le subyace una intención generalmente mora- 
ližante (en el buen sentido): no escribo-describo, doy testimonio de lo real para hacer saber-cambiar. Esta tendencia al acontecimiento histórico, social o natural, comparte una visión rígidamente dual y piensa lo real en bloques sociales globales planteados, desde luego, en oposiciones: lo indio/lo blanco/lo mestizo, el obrero/el burgués, etc. El personaje, el individuo, es pensado como parte o representación de uno de estos bloques y por lo general se inserta en un orden/desorden que lo rebasa, sea éste de índole natural o social. Esta escritura produce un personaje inmerso en fuerzas que no ha «domesticado» y que describen el desgarramiento y el fraccionamiento de un espacio anterior a una ley reguladora que organice los poderes sociales de otra manera.

De todos modos, comparte un gesto social comprometido con el entorno; pero sería erróneo pensar en una «representación» propiamente subversiva. Esta escritura, si bien funda una necesidad de orden, éste no se articula, a su vez, en otro orden de diferencias aplicado a todos por igual, sino que tàn sólo reconoce un estado de violencia, al cual está sujeto el individuo, una clase, la raza, etc. (Socavones de angustia [1953], de Ramírez Velarde; Borrachera verde [1938], de Botello; Tierras de violencia [1955], de Alberto Trujillo, son buenas pruebas de ello).

E1 lénguaje, fundamentalmente denotativo, forma simultáneamente un orden y un contraorden, una necesidad y un conflicto, un ejemplo de lo pensable y lo deseable, pero también un modelo de lo convencional e imposible. Si bien restituye a la sociedad el paisaje, su naturaleza no restituye al hombre su lenguaje; dèsde luego, no me refiero a formas «regionales» del habla, sino a que este lenguaje no es «recordante», no incorpora una historia "profunda», la que hizo y hace posible la sobrevivencia y no la que produce la muerte, por decirlo de algún modo. $\mathrm{Y}$ todo esto al margen de una valoración sobre la obra misma.

En una cantidad considerable de obras no existe un «héroe civilizador». La tensión entre una cultura ignorada pero cotidiana y una cultura reivindicada pero lejana, un mestizaje esperado pero rechazado, perfila un lenguaje que connota tan sólo una parte de lo social: un espíritu ético que incorpora una distancia doble, primero a través de un narrador omnipresente y luego a través de un personaje «explorador»; por tanto, un observador.

El espacio literario del acontecimiento tuvo un primer ciclo, llamémoslo por eso tradicional. Ciclo que podría articularse a través de obras que son logros importantes en nuestra historia literaria; nombraré sólo algunos: Raza de bronce (1919), de Alcides Arguedas, la primera aproximación al mundo indígena; Aluvión de fuego (1935), de Oscar Cerruto, cuyo gesto desborda la simple «copia» para enmarcarse dentro de una te- 
sis política socialista y cuyo desborde prefigura la dinámica social futura, el rol del proletariado minero como líder del movimiento obrero boliviano: Sangre de mestizos (1936), de Augusto Céspedes, en la que la realidad y la ficción se fundan mutuamente ( $E l$ pozo) y realzan dramáticamente el precio que está obligado a pagar un país para poder concebirse a sí mismo, para poder nombrarse.

Esta literatura, que en su gesto primigenio denota más bien un gesto activo respecto a lo real, culmina por la década de los setenta en un «estado pasión» (los términos se invierten: de lo activo se pasa a lo pasivo). Esta inversión, a mi modo de ver, es la señal de la eclosión final de este ciclo que, sin dificultad, puede articularse a la dictadura y a los sistemas de represión. El lenguaje declara su ruptura consigo mismo. El contrato entre significado y significante del signo se rompe. El pacto entre palabra y referente se quiebra. La relación está regida por lo arbitrario. Esta situación límite, importante en toda literatura, señala, por una parte, el final de una relación estimada de hecho (significado-significante = ciudadano-Estado), y por otra, funda la necesidad de encarar de otro modo el lenguaje. $Y$ eso es lo que va a suceder en nuestra literatura actual. Lo veremos más adelante.

Sería erróneo suponer, sin embargo, que aquí acabó la literatura de acontecimiento. De ninguna manera manera. Una historia violenta de dieciocho años de dictadura militar, con el horror que para nadie es desconoeido, sino más bien compartido; la traumatizante experiencia de las guerrillas y la muerte del «Che»; una considerable cantidad de golpes de Estado, además de innumerables desastres sociales, se suman para hacer sentir el peso de una realidad que, histórica, social, económica y culturalmente, va a nutrir a esta literatura. Pero ya será otro el principio organizativo de esta literatura actual de acontecimiento; ya volveremos sobre ello. Por el momento, deseo aproximarme a esa segunda vertiente, a la que lla-: mamos de emancipación.

La literatura de emancipación se opone a la de acontecimiento no necesariamente por el peso de la función referencial. Lo decisivo para esta. distinción es, sobre todo, la actitud respecto a lo real. Si como habíamos: observado la primera es pensada en términos de un catastrófico fatalismo respecto a lo real, la literatura emancipada —y por eso el nombre- más bien elabora por encima de esa sujeción la posibilidad de una manipulación de lo real y que en la literatura actual sustituye el valor de un deber ser sobre el ser, lo moral sobre lo estético.

Quiero centrar el análisis de esta literatura en torno a tres obras, las que en sentido amplio considero como muestras de una primera literatura emancipada. Se trata de la obra de Arturo Borda (1883-1953), Carlos: 
Medinaceli (1898-1949) y Jaime Sáenz (1921). Si los dos primeros autores pueden ser considerados como de tránsito hacia una nueva literatura, la obra de Sáenz es parte de la literatura actual, y es en ese espacio donde ella alcanza su plena realización.

La Chaskañawi (editada en 1947, pero escrita veintitrés años antes), de C. Medinaceli, es tal vez la primera obra que se realiza bajo un signo afirmativo. El personaje, sumido originalmente en el tedioso sinsentido de la alta sociedad chuquisaqueña, se «enchola» al ligarse con una mujer de pollera que lo orienta hacia una vida activa y «progresista». Este acontecimiento literario, audaz e impensable para la pseudoaristocrática elite criolla, infringe las estrictas reglas endogámicas de ésta. Medinaceli, abiertamente, propone el mestizaje en tanto que fuerza fundadora. La Chaskañawi establece un puente entre el pasado y el presente. Como dice L. H. Antezana (Elementos de semiología, La Paz, 1977): «Adolfo (el protagonista) es un tipo del pasado - fin de raza, sin porvenir-; Claudina, la chola, es el presente, que contiene en potencia el porvenir.» $Y$ se trata de un porvenir concreto y no mediado por un desastre y que pertenece a lo que Medinaceli llamó «la búsqueda de lo territorial», que reivindica una libertad interior, aquello que entrega, y esto será uno de los determinantes de la literatura de emancipación, un «gobierno» (J. Sáenz), una «salud», en términos de Antezana. Es necesario subrayar que por primera vez en la novela boliviana el héroe protagónico no muere, no pierde, no es mártir de una causa, sino que, adánico y feliz, salta por encima de su sumisión a lo real, a la que me referí anteriormente que domina a la literatura de acontecimiento.

El loco (obra escrita durante casi toda una vida y recién publicada en 1966), de Arturo Borda, es una de las obras más controvertidas y menos conocidas de la literatura boliviana. Quizá sus tres volúmenes (1.600 páginas) son un reto difícil para cualquier lector. Escritura desigual y dispersa, genéricamente se aproxima más al estilo de un diario de escritor. De todos modos, pienso que es una de las obras más importantes de la literatura boliviana en la medida en que prefigura lo que podría ser en el futuro una literatura boliviana andina.

Borda es, sobre todo, un visionario, al modo de Blake. Un espíritu que hizo la apología de la libertad y que se negó a someterse a todas las convenciones. Escribir viene a ser para él una elección política y ética, y quizá por ello necesariamente marginal.

La narración en El loco avanza en cadena entre silencios, sueños, rupturas y retornos; y cada eslabón es parte de una intención de representación visual de cada una de las situaciones. Si la forma, según Borda mismo, está determinada por el contenido, cada situación imaginada corresponde 
al género elegido. El cambio de lenguaje así efectuado es parte de una constante preocupación por la escritura, que no quiere decir directamente de lo real (exceptuando los ensayos), sino producir una realidad escritural en sí. Lo literario es, en ese sentido, un «efecto», una composición totalizadora. No es sorpresivo, pues, que sea el primero en la literatura boliviana que hace cuentos fantásticos de primera calidad. También son novedosos los personajes que circulan por su obra, que por primera vez en esta narrativa son individuos de ciudad, de las zonas intermedias. Si bien la ciudad nunca está tematizada explícitamente, los moradores están cercados de ciudad: solitarios, mendigos, brujos, prostitutas, locos y sabios indígenas, habitantes de conventillos y bodegas. Pero conventillos y bodegas no son objeto de descripción, sino que simplemente forman parte de un habitat, son sus condiciones concretas.

Pero la intención más importante de la escritura de Borda es la destrucción. Destrucción de formas, géneros, normas y, sobre todo, de valores; trátese del arte o del mundo burgués. Esta escritura demoledora puede, sin forzar, ser entendida como parte del pensamiento andino con relación al milenarismo. El mundo está «al revés» y la destrucción es poner en obra un «Pachakuti», el paso de una edad a otra, que es en lo andino, como en la obra, un cataclismo cósmico. Pero esta destrucción se hace imprescindible para la regeneración. Es, pues, el paso de un valor negado por otro: el comienzo.

Este cambio - $\mathrm{y}$ esto es lo importante- puede ser producido, y es en esa intención que se inscribe la obra de Borda, sea ella pictórica o literaria. A través del arte existe la posibilidad de acción sobre el mundo, y en Borda la acción es la escritura. La metáfora de la muerte de la armonía por el triunfo del arte y el acabamiento general de los valores «blancos» para instaurar un nuevo orden "americano» son un acto de regeneración. En esta perspectiva, el héroe de la obra no es en realidad el personaje, sino el autor.

Borda, antihispanista, comparte con Medinaceli un americanismo profundo, y por eso no sorprende el hecho de que su seudónimo fuera Calibán.

Jaime Sáenz (1921), cuya obra puede señalarse como una de las más grandes actualmente, libera la poesía del pseudomodernismo y del intimismo. Como pocos lenguajes, el de Sáenz — como el de Vallejo- adquiere una voz, una sintaxis propia $o$, si se quiere, una gramática poética que es inconfundible.

Lenguaje simplificado, casi cotidiano podríamos decir, cuyo léxico no es abundante, pero que ha tenido el vigor de transformarse en el contexto 
poético, adquiriendo sentidos complementarios o revelando los sentidos ocultos de las palabras.

Estos tres autores, de modos diferentes, convierten -explícitamenteel espacio literario en el espacio de su vivir, en el espacio de su sentido. Así, el lenguaje está signado por una vitalidad que no reproduce, sino produce, que no «copia», que «elabora». Es por esta razón que se puede hablar de literatura emancipada, en sentido de elaboración, de síntesis, de «efecto».

Este lenguaje, pues, es portador, por lo menos virtualmente, de una reflexión sobre la relación lenguaje-sociedad, individuo-acción.

Esta literatura emancipada hace de la literatura un acontecimiento, o el acontecimiento, y en ese sentido, y junto a las renovaciones técnicas que aparecen junto al boom latinoamericano, señalan el camino que va a seguir la nueva literatura boliviana.

Ahora bien: la literatura de acontecimiento, por su naturaleza «fatalista», está más cerca de la tragedia que del mito. Ella instaura una historia de cerca, presentada no sólo en términos de verosimilitud, sino como verdad, precisamente en contra del mito y de la epopeya, aun si intenta fundar una utopía. Esta literatura rechaza la ilusión y propone asimilar lo real vivido como parte de la lucha social de clases. En ese sentido se presenta como testimonio.

Esta tendencia en contra de la ficción y en favor de un relato de lo real se intensifica al extremo de cuestionar el fundamento mismo de la literatura: el lenguaje, la fe en la posibilidad de comunicar, su eficacia en un mundo en que la palabra deja de tener sentido, sustituida como está por la violencia, el silencio o la sospecha.

Las obras que expresan esta ruptura deben ser consideradas como un cuerpo literario de transición hacia lo que sería el nuevo espacio escritural. Quiero referirme sólo a tres libros.

La novela de Marcelo Quiroga Santa Cruz, Los deshabitados (1957), considerada por la mayor parte de los críticos como la novela de transición, corresponde temáticamente a esta ruptura. Los seres aparecen reducidos, en el lenguaje, a sólo la función de contacto o fática. Se apela al significante para confirmar presencias reiterando significantes. Los personajes de la novela, «vampiros» que se nutren unos de otros, rota la posibilidad de articularse de otra manera a la sociedad y al mundo, culpables y mezquinos, solitarios, se cubren de silencio y encubren su violencia. Son personajes vacíos de lenguaje. Durcot, el escritor, no escribe nunca.

Esta ruptura señala también otra tensión: la imposibilidad de pensar 1a escritura en relación a la sociedad. En ese sentido marca una impotencia.

Estrella segregada (1973) es, probablemente, la obra más perfecta y lú- 
cida de esta escritura de transición. En ella Oscar Cerruto logra, con claridad, expresar la crisis no sólo de un grupo social unido al poder, sino también, paralelamente, la pérdida del lenguaje que le era afín. El poema - se trata de varios poemas que configuran en realidad una unidad- se invalida a sí mismo a través de la denuncia del lenguaje convertido en «engañoso» y voluble. Lenguaje que por trocarse en desleal respecto a lo nominado ha perdido toda posibilidad de convocatoria. Sólo la palabra no pronunciada guarda esa lealtad poética, pero cuyo «silencio» pone en duda su propia existencia. Esta pérdida de fidelidad de la palabra inaugura un texto del terror regido por un sistema jurídico-lingüístico cuya ley abstracta se realiza en efectos de culpa-castigo, de soledad y de silencio.

Esta ruptura del contrato entre palabra y sentido expresa el reino de lo caótico; todas las certezas desaparecen, a no ser la de la muerte. Todas las seguridades del lenguaje se tornan sospechosas, y en esa perspectiva no puede existir posibilidad de comunicación. Queda, pues, un significante sospechoso, una presencia recelosa, cuerpos yuxtapuestos, cuyas tramas no se identifican.

En Estrella segregada el poeta es vencido por «los de abajo», por su peso insoportable e insobornable, por la ciudad que lo habita negativamente porque no corresponde a su deseo: Todo quiso ser / nada ha sido.

También otra novela de importancia señala esa victoria de lo real condenando al individuo a una escritura «imposible». Se trata de Morder el silencio (1980), de Arturo von Vacano. En ella, la Bestia (el poder de la represión) triunfa sobre el escritor, que, encarcelado y exiliado, se autocondena a no superar la amenaza de la «Bestia», probando así que efectivamente no existen salidas.

La ruptura de la relación entre escritor y lenguaje es marca de un quiebre más profundo. Los autores han dejado de pensarse en términos comunitarios, en bloques sociales, en dualidades. Un proceso de individuación evidente transforma su relación con lo social. Se han quedado solos con su palabra, que ha cesado de ser acción. ¿Cuánto tiene que ver este proceso con los resultados de la revolución de 1952 ? No es gratuito que en vida Cerruto se encierre en su soledad ni que, como contrapartida, Marcelo Quiroga se lance a la política (del partido socialista), que le cobra la vida, ni que $V$. Vacano se quede en el exilio. Si la palabra ha perdido su poder activo, se ha separado de lo colectivo, no queda más remedio que asumir la tarea de escribir bajo otra luz. Así, la literatura tradicional, ligada fundamentalmente a una ética social, al «deber ser», se transforma en la nueva literatura emancipada al «ser», es decir, a una escritura cuya 
moral se remite a ella misma. Volveremos sobre ello. En tanto que en la literatura de acontecimiento, si bien subsiste este «deber ser", éste se remite directamente al autor: yo debo escribir así porque estoy en camino hacia el hombre (revolucionario) nuevo. Escritura y protagonista son parte de un proceso de transformación, es decir, remiten a una conciencia del autor-individuo.

Si la literatura de transición marca de algún modo una clausura, ella permanece en algunas formas. Las nuevas representaciones y territorios son interiores: las moradas, las casas, los cuartos. La forma dialógica, y entre ellas sobre todo el monólogo, casi la confesión, parece ser uno de los estilos preferidos. Ciertamente, el diálogo puede articularse con la intimidad de estos interiores.

En cuanto a la nueva literatura de acontecimiento, aparece una literatura clandestina, en términos de abierta rebeldía en contra del sistema de represión, pero secreta en su tono confidencial, solitaria en su monólogo interior. Esta forma de confesión garantiza la honestidad y la verdad del discurso, que no deja de tener una fuerte carga religiosa cristiana. Si el gesto de esta literatura es también persuadir y convencer, también es de algún modo corresponder. Es así como estructuralmente se articula a un sentido solidario que toma al otro como punto de referencia. En ese sentido, la escritura se dice «prójima», individualmente ética.

Es indudable que las nuevas formas dialógicas y un renovado intento de mimesis entre el lenguaje hablado y escrito dan cuenta de la pluralidad social boliviana. En ese sentido, la literatura boliviana intenta ser globalizante.

Ya en la nueva novela indigenista «campesina», aquella que se origina después de la Reforma Agraria (1952: fin del latifundio, expulsión de los patrones, fin del régimen de servicios personales obligatorios), se establecen los primeros «contactos» entre campesino e hijo de patrón ( $E l$ valle del Cuarto Menguante [1975], de Boero Rojo). Las relaciones han cambiado, y como dice Antezana, «otros son los enemigos para el campesinado y otros los aliados — como los mineros- en vista de una lucha más amplia, lejana ya de la tradicional lucha por la tierra propia o contra los abusos del patrón» (Antezana, «Tendencias de la narrativa boliviana», en prensa).

Cabe anotar, sin embargo, el riesgo de una literatura que redunda en el acontecimiento. $Y$ éste es el esquematismo de una lógica narrativa $y$, por qué no decirlo, poética reiterada. Si no hubo novela indigenista sin violación, si no hubo novela minera sin masacre final (J. Sanjinés lleva ambas «lógicas» al cine), ahora existen tres cuentos de tres autores distintos que reproducen una misma estructura narrativa; se trata de la desapa- 
rición de los «revolucionarios» fondeados en el lago Titicaca y, en un caso, arrojado al Illimani. Raúl Leyton, en Chakani; C. Verdúguez, en Un grito en el silencio (la mejor versión, sin duda alguna), y R. Poppe, en María. Esta misma tendencia está presente en las mismas antologías que circulan sobre la nueva narrativa boliviana: Don Quijote y los perros (Antología del terror politico), de Néstor Taboada Terán, y Los golpes, de Adolfo Cáceres.

Pareciera que un esfuerzo de "realidad» dominara sobre lo literario. Así, las dimensiones míticas y fantásticas son poco elaboradas en el espacio literario. Tal vez porque el mundo cotidiano está abundantemente provisto de dimensiones míticas, tal vez porque los acontecimientos sociales no dan lugar a la ficción, tal vez porque el discurso oral ha demostrado ser más adecuado y vigoroso para ese fin, por su posibilidad de ser inmediato, quién sabe...

Una naciente dimensión mítica se muestra en la narrativa minera de René Poppe. Unida a esa interioridad a la que me he referido antes -es casi por primera vez que en la literatura minera se pasa del «exterior» al «interior mina»- articulada también a un espacio clandestino, es una escritura abiertamente politizada, pero a la vez, dotada de un lenguaje minero que establece una clausura, un código no comprensible por cualquiera y que otorga a la narrativa de Poppe un doble sentido: por una parte, concuerda con una vida que participa del mundo de las sombras míticas junto al «tío», personaje favorable pero también temible - y que de hecho los hace socialmente clandestinos- y la luz, enemiga, porque está habitada por la muerte, encarnada en la violencia del poder. Y, por otra parte, esta experiencia intermedia se articula al rol de primer orden que tienen los mineros en la política boliviana.

Es evidente que la Revolución Cubana y particularmente todo el movimiento de la guerrilla dieron origen a un nuevo entusiasmo entre los intelectuales y artistas latinoamericanos. En Bolivia, esta escritura, paradigma de una actividad religioso-cristiana, puede ser considerada, en palabras de Javier Sanjinés, como «un viaje hacia el hombre nuevo». Si bien ligada al acontecimiento histórico de modo inmediato, no será éste el que es cuestionado. Lo importante es el trazo espiritual del hombre, su capacidad de entrega, su acto de fundación, que es participar en una causa común: la patria nueva. Dos ejemplos: Los fundadores del alba (1969), de Renato Prada, una de las primeras obras de la literatura de guerrilla. E1 protagonista, en un juego de tiempos con el narrador, da cuenta, a través 
de monólogos íntimos, de un proceso que se quiere transfigurativo. Esta continuidad subjetiva da la pauta, que no es lo «exterior»-la lucha-, lo que determina la organización del texto, sino la formación interior, garantía de un cambio que a la larga apunta al «hombre nuevo», el único que puede garantizar una transformación verdadera del mundo. El lenguaje es testimonio de este «camino» y al mismo tiempo su realizador.

Algo parecido sucede con el libro de Pedro Shimose Poemas para un pueblo (1968). Aquí el autor asume al hombre nuevo, cuya tarea consistirá en efectuar una nueva nominación del territorio patrio como signo de su voluntad de integración nacional, como señal de su solidaridad. De ese modo, la palabra es origen, idea, que ya A. Céspedes expresó en Sangre de mestizos: «Lo que se hizo y no se dijo no ha existido». Nombrar equivale, entonces, a nacer; nombrar es fundar una nueva conciencia, un nuevo modo de hacer la realidad. $Y$ en esa intención se inscribe este libro de poemas al hacer un recorrido verbal por todo el territorio patrio para convertirlo así en una nación desalienada y consciente de sí misma.

El proceso de individuación se interioriza de tal manera que termina por afectar al discurso verbal mismo en sentido que el acontecimiento se traduce en su formación. En Quiero escribir pero me sale espuma (1972), también de Pedro Shimose, el discurso de la violencia invade las formas mismas que lo sostienen.

Los espacios sintácticos ya no son respetados, las palabras se aglomeran, pierden su autonomía gráfica y el lenguaje se vicia y degrada por formas ajenas que alienan hasta confundir el código: American way of life es un buen ejemplo. La ironía y la paradoja hirientes son los nuevos elementos junto a un tono coloquial que, si no está enredado con una intención agresiva, funciona en estructuras populares, sea en idiomas indígenas o traducidas al español. Estos últimos son el gesto de las solidaridades.

Esta nueva forma de violencia, asumida esta vez por lo verbal, está articulada a ese proceso de interiorización en el lenguaje como signo transportado independientemente de lo dicho. Este es uno de los «encuentros» más decisivos de la nueva literatura. Ahora, la formación verbal, en los aspectos señalados, acompañan el significado o, como en esta última obra, lo realiza el significado.

Es notable cómo gran parte de la generación más joven ve en estas formas - en la voz dialogal, en lo coloquial, en el lenguaje directo-, a pesar de las rupturas, de la ironía y el sarcasmo, la posibilidad de enfrentar un sistema autoritario. Esto, por lo demás, no es particular de Bolivia, sino probablemente una forma latinoamericana.

Hasta aquí en la literatura de acontecimiento, respecto a la que decíamos «tradicional», se ha efectuado un proceso de interiorización que va 
desarticulándola del acontecimiento, desplazando al suceso dentro del lenguaje, que se convierte así en el símil de lo real: la violencia social violenta a su vez las formas del lenguaje. Sin embargo, su cordón umbilical, aquello que la define desde una visión más bien «fatalista» - lo «real» por encima de lo «imaginario»-, la reiterada aproximación a una utopía marcada por la muerte del héroe, la ética de un «deber ser» individual o colectivo, permanece fuertemente arraigada y vincula esta literatura a un comportamiento social moralizante (en el buen sentido).

Más profundo es el cambio en la literatura emancipada, que en el caso del primer Sáenz, Borda, Medinaceli contenía un proyecto, un comportamiento social o individual incorporado al discurso, ahora sencillamente se deja ser: es. Se podría hablar, entonces, de una doble independencia. Por una parte, se desliga de un proyecto individual (Sáenz) o social (Medinaceli, Borda), y con ello se desliga de un discurso ético. Por otra, como en la tradicional, comprende a la literatura como una totalidad que no hace uso del referente exterior para cumplirse. Estas dos «libertades» abren un imaginario nuevo que, a mi modo de ver, se puede entender como subversivo con respecto a la literatura anterior, porque se da el derecho de habitar el mundo sin mediaciones represivas acudiendo simplemente a lo que es.

La tendencia de incorporar en el juego verbal el sentido o sinsentido ya había tenido en Jaime Sáenz un exponente extraordinario. Algunos poemas, pero sobre todo las Memorias, en la novela Felipe Delgado (1980), alcanzan un humor indiscutible. E1 juego de palabras, versiones, inversiones, retruécanos, simplificaciones, la inconsistencia del significado instauran un alegre discurso del sinsentido pleno de humor. También, aunque en otro sentido, El otro gallo (1982), de Jorge Suárez, realza la función de la palabra en su poder imaginativo, de apertura, en su poder de escuchar/se y vivir en una esfera de mentira alegre.

Convertir el lenguaje en un acontecimiento pleno de sugerencias tiene en el Manchay Puytu (1979), novela de Néstor Taboada, un ejemplo hermoso y novedoso, en el sentido que llega a asumir en la narración el lenguaje y el ritmo de la lengua quechua yuxtapuesta en el texto, simplemente, al español, en función de acompañante. Es de las pocas novelas que se remiten a un pasado colonial. En ella, Taboada intenta reconstruir el choque ideológico entre una cultura andina y la española a través de una leyenda popular: el Manchay Puytu, que relata los amores de un cura indígena y su empleada india.

En los poemas de Eduardo Mitre esta tendencia adquiere todo su «esplendor». Morada (1975) y Mirabilia (1980) son poemas celebratorios de lo cotidiano, en los que el lenguaje intenta mostrarse a sí mismo y los 
poemas vienen a ser «objetos» independientes, en realidad, de su referente para ser ellos en sí mismos. Las palabras orientadas a partir de su polisemia abren a través del texto dos o más espacios de lectura. La valorización de la página en blanco y la síntesis prestada a los haikus colaboran a una poesía concreta y espacial de primer orden.

Finalmente, quiero citar a Jesús Urzagasti, novelista y poeta, en quien, de algún modo, intervienen algunos de los aspectos que venimos anotando: la mirada sobre el lenguaje y la tendencia dialógica.

En la novela corta Tirinea (1969) una compleja estructura de narradores - voces - se multiplican para dar cuenta del personaje que, a su vez, escribe un relato autobiográfico: el personaje -Fielko- «se desdobla en múltiples Fielkos, creando así una pluralidad de narradores personales» (Antezana, 1983). También en El país de I papecuana, novela inédita, tres narradores «arman» la novela: el Rural, el Intelectual y el Muerto. Urzagasti, chaqueño, re-conoce -me parece que es el único en la literatura boliviana - un pasado mítico como fundamento de su escritura: la selva (esa misma selva que nutrió nuestra literatura después de la Guerra del Chaco, pero ide qué otro modo!) como espacio anterior, mítico, que reproduce las fuerzas simbólicas y que hacen posible a la poesía como sentido de armonía y unidad. La naturaleza, un leit-motiv tan reiterado en nuestra literatura, adquiere aquí otro sentido y en contra del sinsentido, asegurando de ese modo la armonía efectiva con el mundo.

De ahí que la escritura ya no es reconocida en su sola discursividad -horizontalmente-, sino en su profundidad, en su don: entrega la posibilidad de habitar el mundo.

Ahora bien, ¿qué es lo que se puede resumir de esta nueva literatura? En términos generales, este espacio, a partir de la revolución de 1952, expresa una especie de proceso de individuación. Las grandes multitudes que habitaban nuestra literatura «tradicional» han quedado desplazadas por individuos localizables en las clases medias o intermedias. En esa perspectiva podríamos anotar que si el lenguaje era «mandarín», ahora es cotidiano y coloquial. Toda una articulación - no citada sino sintéticamente- de literatura de acontecimiento se establece con diferencias que, sin lugar a dudas, la relaciona a los acontecimientos políticos y a la experiencia técnica de otras literaturas.

Sintomáticamente es la narración la que se ha hecho cargo de la primera persona, la que interioriza el acontecimiento rechazando a un narrador omnipresente o alternando con él, para fijar con mayor facilidad y 
verosimilitud el lenguaje en su medio social. En tanto que la poesía tiende más bien a abandonar la primera persona y, encubierta en una tercera, busca dar mayor «objetividad» al poema.

Pero fundamentalmente es el espacio literario el que, pienso, ha cambiado; más bien, la reflexión sobre éste, la elaboración que exige, el modo de concebirlo. Podemos reconocer varias «versiones» sobre este espacio: por una parte, ya lo vimos, registra la vida, pensada en presente (Borda). La escritura como vía ascensional, recorrido interior, en Sáenz: toda su obra poética lo demuestra, y dicho en sus términos, se trataría de recorrer esta distancia, una especie de transformación interior próxima a una experiencia mística; hablaríamos aquí tal vez de una transfiguración literaria siempre y cuando esté a salvo de la idea de ficción, puesto que lo imaginario es una forma de conciencia tan válida como cualquier otra. En Sáenz la escritura funda un paralelo con la vida con la cual intenta fundirse. La equivalencia escribir = vivir (si la entendemos como la interioridad de la vida) adquiere todo su sentido. En cierto modo, también en Renato Prada y Pedro Shimose la escritura registra el comportamiento del «hombre nuevo», es también un «viaje ascensional», una manera de transfiguración.

Por otra parte, el espacio es concebido como morada y como puente hacia la vida, asegurándole ámbito y un sentido en el mundo.

En resumen, existen, en abundancia, obras que ya no conciben el espacio literario como comunicación testimonial, ni aun, paradójicamente, aquella literatura de la guerrilla, en la cual precisamente lo importante, como habíamos señalado, es la conquista del hombre nuevo. Nombrar es transformarme.

Individualidad e interioridad son signos de una reflexión que convierten a la palabra en acontecimiento. La literatura se ha vuelto atenta hacia sí misma. Es cierto que tal vez este cambio de atención esté articulado con una sociedad que niega los valores individuales y que no ha mostrado tener alternativas respecto a su propia historia social. Exaltar, entonces, la literatura, celebrarla, no es sino un modo de celebrar lo simple, lo gratuito; pero también un sentido, una plenitud, una identidad encontrada al mundo. Y, por lo mismo, puede ser considerada como un acto de rebelión subversiva, en la medida en que implica una lealtad al sentido, un gesto de lealtad a la vida y a lo vivo.

¿Qué significa esta doble desarticulación con lo que llamamos al principio de este trabajo el proyecto de la literatura boliviana? Pienso que, en lo que respecta al mundo castellano-boliviano, el proceso de identificación que se expresa como conciencia nacional ha cesado. Esto significa enton- 
ces haber encontrado una armonía: somos lo que somos, y nos expresamos no con el deber, sino con el deseo. $Y$ en lo que se refiere al lenguaje, está evidentemente inmerso en un proceso de mestizaje lingüístico, que se traduce formalmente en la pluralidad de las voces, en la violencia de los encuentros. Todo esto en concordancia profunda con una memoria que se va haciendo social y literariamente y que, a mi modo de ver, a estas alturas ya se manifiesta con rasgos específicos. Pero esto ya es otro cantar.

La Paz, Bolivia, agosto de 1985. 